\title{
Primary Cardiac Rhabdomyosarcoma in a Child-A Case Report
}

\author{
Krishna Prasad Maram¹, Vikram Kudumula², Dilip Ratti ${ }^{3}$, Sindhura Paturi ${ }^{1}$ \\ ${ }^{1}$ Department of Pediatrics, Andhra Hospitals, Vijayawada, India \\ ${ }^{2}$ Department of Pediatric Cardiology, Andhra Hospitals, Vijayawada, India \\ ${ }^{3}$ Department of Pediatric Surgery, Andhra Hospitals, Vijayawada, India \\ Email: maramkp@gmail.com
}

How to cite this paper: Maram, K.P., Kudumula, V., Ratti, D. and Paturi, S. (2021) Primary Cardiac Rhabdomyosarcoma in a Child-A Case Report. World Journal of Cardiovascular Surgery, 11, 17-20. https://doi.org/10.4236/wics.2021.112003

Received: January 1, 2021

Accepted: February 23, 2021

Published: February 26, 2021

Copyright (c) 2021 by author(s) and Scientific Research Publishing Inc. This work is licensed under the Creative Commons Attribution International License (CC BY 4.0).

http://creativecommons.org/licenses/by/4.0/

\begin{abstract}
Unlike benign primary cardiac tumors like rhabdomyomas and fibromas, primary malignant tumors are extremely rare in children with very few cases reports in the literature so far. Rhabdomyosarcoma is a rare primary malignant tumor in children with most reported cases occurring in the right heart. We report a 15-month-old child with a primary rhabdomyosarcoma arising from the left ventricle and presenting with cardiac tamponade and circulatory failure. He underwent a diagnostic sternotomy and palliative chemotherapy but succumb to the disease later.
\end{abstract}

\section{Keywords}

Rhabdomyosarcoma, Pericardial Effusion, Sternotomy

\section{Introduction}

Cardiac tumors in children are rare, with the majority being benign primary tumors like rhabdomyoma and fibroma. Malignant tumors of the heart are extremely rare in children, with sarcomas topping the list followed by lymphomas. However, unlike adults, metastatic cardiac tumors are less common than primary malignant tumors of the heart in children. Children with cardiac tumors can be asymptomatic or may present with chest pain, murmur, arrhythmia, heart failure, or sudden death, depending on the location and size of the tumor [1]. We report a very rare case of primary rhabdomyosarcoma of the heart presenting with a life threatening pericardial effusion, and massive local tissue infiltration.

\section{Case Report}

A 15-month old boy had presented with fever and respiratory distress of three 
days duration. At the time of admission, he had signs and symptoms of acute circulatory failure with tachypnea, tachycardia, elevated Jugular Venous Pulse and hypotension. He was febrile and irritable, but had no rash. Chest auscultation showed good bilateral air entry but muffled heart sounds. Abdominal examination was otherwise normal except for hepatomegaly. He had no significant history of illness prior to the current admission. He had normal growth and vaccinated as per recommended schedule. His Chest X-Ray (Figure 1) demonstrated significant cardiomegaly and a bedside 2D-Echocardiogram (Figure 2(b)) showed a massive pericardial effusion causing an impending tamponade, and a solid vascular mass in the left ventricle extending into the anterior as well as superior mediastinum. In view of hemodynamic instability due to cardiac tamponade, he underwent an immediate needle pericardiocentesis which drained around $200 \mathrm{ml}$ of blood stained fluid followed by symptomatic relief of respiratory distress. A smear study of the pericardial fluid showed presence of benign reactive mesothelial cells, macrophages and neutrophils but had no evidence of malignancy. In light of 2D Echocardiography findings and diagnostic dilemma, it was decided to go for a surgical evaluation of the cardiac mass.

A midline sternotomy revealed a large lobular mass (Figure 2(a)) arising out of the left ventricle, infiltrating the myocardium and extending into the anterior

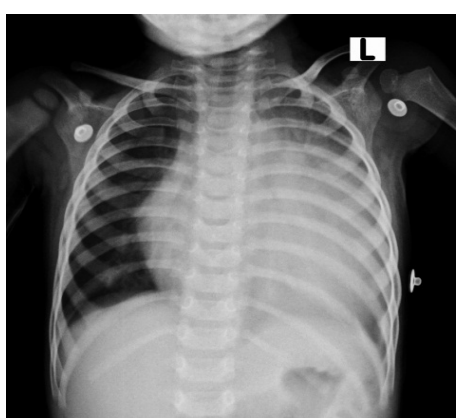

Figure 1. Chest x-ray showing massive cardiomegaly.

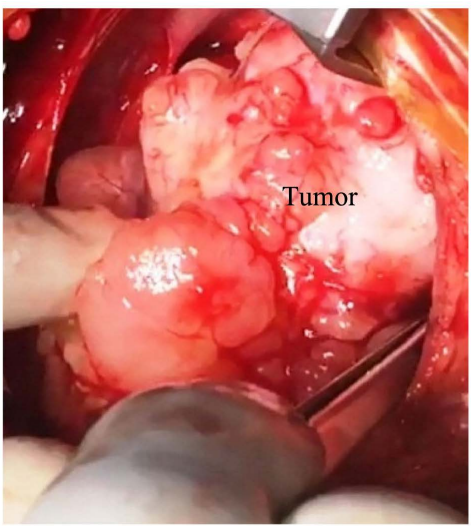

(a)

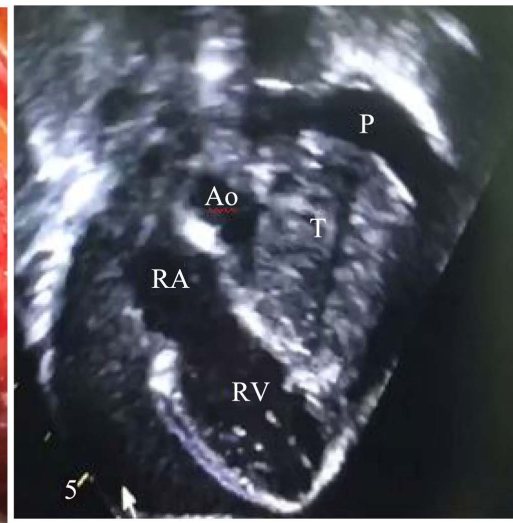

(b)

Figure 2. (a) Intra-operative picture of the cardiac tumor; (b) 2D-ECHO showing tumor arising from LV. T = Tumor, $\mathrm{P}=$ Pericardial effusion, Ao = Aorta, LV = Left Ventricle, $\mathrm{RA}=$ Right Atrium. 
and superior mediastinum. Due to the extent of local tumor infiltration, it was considered inoperable. The biopsy of the mass showed a tumor with intermingled dense, compact cellular and loose myxoid areas. The cellular areas are predominantly seen around dilated blood vessels, and composed of sheets of small spindled to round cells with hyperchromatic, round nuclei. The biopsy and immunohistochemistry analysis were consistent with a diagnosis of spindle cell rhabdomyosarcoma. Further imaging to delineate the extent of tumor infiltration was not done due to lack of parental consent and financial constraints. He underwent palliative chemotherapy with VAC regimen (Vincristine, Dactinomycin plus Cyclophosphamide) but succumbed to the disease six months later.

\section{Discussion}

Primary malignant tumors of the heart are extremely rare, constituting around $10 \%$ of cardiac tumors in children. Sarcomas are the most common primary cardiac malignant tumors (95\%) followed by lymphomas [1]. Angiosarcoma, rhabdomyosarcoma and undifferentiated sarcomas are the most common sarcomas in children in the order of frequency of occurrence [2].

Rhabdomyosarcomas are mesenchymal tumors originating from undifferentiated striated muscle. They are more common in males [3], and show extensive and aggressive local tissue infiltration and vessel extension [4]. These tumors tend to be single and can involve any heart chamber. Our case had a tumor origin from the left ventricle, though most reported cases had a tumor arising from the right heart. In 45 cases of rhabdomyosarcoma reported in the literature, 17 cases were arising from the right ventricle and 16 cases had involved left atrium, and right atrium in 12 cases [5].

Histologically, they are classified as embryonal, alveolar, pleomorphic and other types while embryonal variety (75\%) is the most common type in pediatric population [6]. Most cases of rhabdomyosarcomas are sporadic in nature although association with familial syndromes like LiFraumeni and Neurofibromatosis type I is not uncommon [7]. The clinical presentation could include cyanosis, signs and symptoms congestive heart failure, chest pain, outflow tract obstruction, recurrent pericardial effusions, arrhythmias, pulmonary emboli and rarely sudden death depending upon the size and location of the tumor. A detailed Echocardiography or Computing Tomography (CT)/Magnetic Resonance Imaging (MRI) of the heart helps with establishing the diagnosis. Open surgical or endomyocardial biopsy aids the histological diagnosis of the tumor. A thorough search with CT/MRI must be made to ascertain the metastatic spread of the lesion. Palliative debulking surgery, chemotherapy and radiotherapy extend survival but prognosis is extremely poor with short survival (upto 13 months) due to local infiltration and metastasis [8].

\section{Conclusion}

Primary malignant tumors of the heart are extremely rare in children. Rhabdo- 
myosarcomas are second most common primary malignant tumor involving the heart and often show extensive local and distant tissue metastasis. The clinical presentation depends on the site of occurrence and commonly presents with cyanosis, arrhythmias, pericardial effusions and outflow tract obstruction. Most of them present late at diagnosis and often not amenable to surgical resection. Despite chemotherapy, debulking surgery, they carry a very poor prognosis with short survival.

\section{References}

[1] Uzun, O., Wilson, D.G., Vujanic, G.M., Parsons, J.M. and De Giovanni, J.V. (2007) Cardiac Tumors in Children. Orphanet Journal of Rare Diseases, 2, 1-14. https://doi.org/10.1186/1750-1172-2-11

[2] Tao, T.Y., Yahyavi-Firouz-Abadi, N., Singh, G.K. and Bhalla, S. (2014) Pediatric Cardiac Tumors: Clinical and Imaging Features. RadioGraphics, 34, 1031-1046. https://doi.org/10.1148/rg.344135163

[3] Roberts, W.C. (1997) Primary and Secondary Neoplasms of the Heart. American Journal of Cardiology, 80, 671-682. https://doi.org/10.1016/S0002-9149(97)00587-0

[4] Nadas, A.S. and Ellison, R.C. (1968) Cardiac Tumors in Infancy. American Journal of Cardiology, 21, 363-366. https://doi.org/10.1016/0002-9149(68)90140-9

[5] Schmaltz, A.A. and Apitz, J. (1982) Primary Rhabdomyosarcoma of the Heart. Pediatric Cardiology, 2, 73-75. https://doi.org/10.1007/BF02265621

[6] Ota, S., Shimonaga, T., Yuki, S., Kuraoka, K., Ogawa, T. and Hirata, T. (2020) Eribulin Therapy for Primary Cardiac Rhabdomyosarcoma. Anticancer Drugs, 31, 304-309. https://doi.org/10.1097/CAD.0000000000000860

[7] Dasgupta, R., Fuchs, J. and Rodeberg, D. (2016) Rhabdomyosarcoma. Seminars in Pediatric Surgery, 25, 276-283. https://doi.org/10.1053/j.sempedsurg.2016.09.011

[8] Burke, A. and Virmani, R. (2008) Pediatric Heart Tumors. Cardiovascular Pathology, 17, 193-198. https://doi.org/10.1016/j.carpath.2007.08.008 